\title{
Referenceless Stratification of Parenchymal Lung Abnormalities
}

\author{
Sushravya Raghunath, Srinivasan Rajagopalan, Ronald A. Karwoski, \\ Brian J. Bartholmai, and Richard A. Robb \\ Mayo Clinic College of Medicine, Rochester, MN, USA \\ \{raghunath.sushravya, rajagopalan.srinivasan, karwoski.ronald, \\ bartholmai.brian, robb.richard\}@mayo.edu
}

\begin{abstract}
This paper introduces computational tools that could enable personalized, predictive, preemptive, and participatory (P4) Pulmonary medicine. We demonstrate approaches to (a) stratify lungs from different subjects based on the spatial distribution of parenchymal abnormality and (b) visualize the stratification through glyphs that convey both the grouping efficacy and an iconic overview of an individual's lung wellness. Affinity propagation based on regional parenchymal abnormalities is used in the referenceless stratification. Abnormalities are computed using supervised classification based on Earth Mover's distance. Twenty natural clusters were detected from $372 \mathrm{CT}$ lung scans. The computed clusters correlated with clinical consensus of 9 disease types. The quality of interand intra-cluster stratification as assessed by ANOSIM R was $0.887 \pm$ 0.18 (pval $<0.0005$ ). The proposed tools could serve as biomarkers to objectively diagnose pathology, track progression and assess pharmacologic response within and across patients.
\end{abstract}

Keywords: Referenceless stratification, affinity propagation, idiopathic pulmonary fibrosis, glyphs.

\section{Introduction}

The disease processes in Diffuse Parenchymal Lung Disease (DPLD) are characterized by distinct cellular infiltrates and extracellular matrix deposition and are broadly classified in CT scans into five primal forms- normal, emphysema, ground glass, honeycombing and reticular. The distribution of these patterns through the lung lobes is indicative of a specific DPLD disease. Differentiability of different DPLDs is central to early application of appropriate therapy so as to positively affect patient prognosis. Existing clinical decision support tools are suboptimal for consistent characterization and visual representation of the type and extent of disease in a clinician and patient friendly manner.

Image based stratification of parenchymal abnormalities specific to patient data in a referenceless manner (with no alignment to a common coordinate system) would facilitate consistent assessment of patient lung wellness. Previously, referenceless unbiased stratification has been proposed for cardiac images 112 . 
No such approach exists for the lung, especially to stratify the biological behavior of abnormalities across patient populations. We address this unmet need using a computational tool to stratify and a visualization tool to provide an iconic overview of the stratifications.

Lung abnormalities were determined in terms of the primal CT appearance forms through supervised classification. During the training phase, radiologists selected multiple volumes of interest (VOIs) to represent the training sets for each of the primal forms. For the supervised classification, earth mover's distance was used as the similarity metric between the histograms of a voxel's neighborhood and the labeled training set. The spatial distribution of the primal forms across the lobes was computed and mapped into glyphs. A new pairwise dissimilarity metric, spikelets, and affinity propagation [3] were used to stratify abnormality distribution across the lung lobes. The clusters were analyzed statistically with Analysis of Similarity (ANOSIM). The exemplars and their respective candidates were automatically categorized into clinical groups using the guidelines in 4 . The glyphs and the underlying classifications were verified by the radiologist to assess correctness. Our experimental results suggest that the proposed tool is a valuable technology to realize the potential of $\mathrm{P} 4$ medicine through direct improvement of quality and consistency in clinical pulmonary practice.

\section{Materials and Methods}

\subsection{Datasets, VOIs and Classification}

CT scans from 372 patients with disease across the DPLD spectrum were used for this study. The patients were scanned on a HRCT scanner $(140 \mathrm{kVp}, 250 \mathrm{mAs}$, BONE kernel recon, $1.25 \mathrm{~mm}$ slice thickness, $50 \%$ overlap with $0.625 \mathrm{~mm}^{3}$ voxels). Three radiologists screened the scans and selected a subset of 14 datasets to pick multiple $15^{3}$ VOIs across the primal forms. 976 VOIs were selected to represent $80,150,187,265$, and 294 VOIs of emphysema, ground glass, honey combing, normal and reticular forms, respectively. The lungs, airways and vessels were extracted using readily available techniques. During supervised nearest neighbor classification, the histogram of $15^{3}$ neighborhood around each parenchymal voxel was compared against the VOI histograms using Earth Mover's Distance.

\subsection{Regional Abnormality Distribution and Glyph Creation}

The lobar regions of the individual lungs were segmented using an approach similar to that described in 5. The distribution of the five primal forms within the respective lobes was computed and used to create the glyphs. Figure 1 shows a representative glyph. The glyph is divided into six regions each representing a lung lobe. The lobes are uniquely labeled to indicate their spatial location. The first letter $(\mathrm{R} / \mathrm{L})$ denotes the right and left lung, the second letter $(\mathrm{U} / \mathrm{M} / \mathrm{L})$ denotes respectively the upper, middle and lower lobes. Although no pleural separation demarcates the lingula from the remainder of the LU lobe, this anatomic region was defined as LM lobe by reflection from the right lung. The origin of 


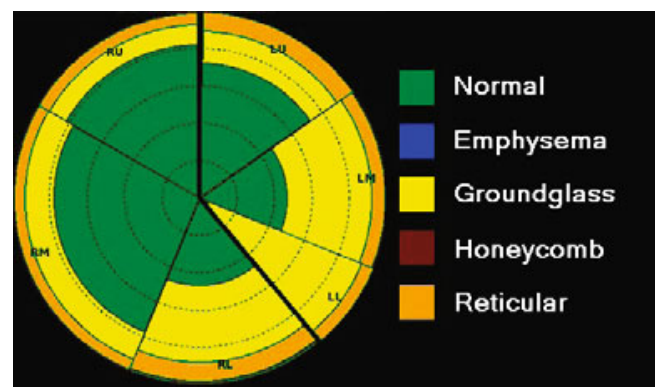

Fig. 1. Glyph layout of the distribution of abnormal patterns across different lobes of the lung

the glyph is fixed at 12-o'-clock starting with the LU lobe. The asymmetry, if any, between the left and right lungs can be readily observed in the glyph. The individual lobes span through angles proportional to their respective volumes. Within each lobe, the disease distribution is represented by color-coded sectors proportional to the percentage of disease in that lobe. The concentric circles are drawn at $20 \%$ intervals for enhanced visualization.

To create an unbiased stratification of the abnormality distribution, affinity propagation [3] - an unsupervised clustering technique that automatically finds the natural number of clusters- was used. Affinity propagation uses message passing to iteratively find clusters from pair-wise dissimilarities of n-dimensional data. In addition to resolving the clusters, it identifies the exemplar that is most 'central' to each of the clusters. Pairwise dissimilarity between given lungs $A$ and $B$ based on their respective lobar distribution of the five primal patterns was computed as

$$
\begin{gathered}
D(A, B)=\sum_{R=1: 6} \alpha_{R} * d_{R}(A, B) ; \quad d_{R}(A, B)=\sum_{i=1: 5} \operatorname{spikelet}\left(A_{i}^{R}, B_{i}^{R}\right) \\
\text { spikelet }\left(A_{i}^{R}, B_{i}^{R}\right)=\left\{\begin{array}{cc}
\frac{\left|A_{i}^{R}-B_{i}^{R}\right|}{\max \left(A_{i}^{R}, B_{i}^{R}\right)} & \text { for } \max \left(A_{i}^{R}, B_{i}^{R}\right)>\text { acuity } \\
0 & \text { otherwise, }
\end{array}\right.
\end{gathered}
$$

where, $\alpha_{R}=100 / \overline{V_{0} l_{R}} ; \overline{V o l_{R}}$ is the mean lobar volume; $A_{i}^{R}$ is the $\%$ distribution of $i^{\text {th }}$ primal pattern in the $R^{\text {th }}$ lobe of lung $A$; and acuity is the minimum resolvable distribution differences, which was set to $2 \%$ in our experiments.

The clusters were categorized automatically into clinical groups using the guidelines suggested in 4]. Accordingly, based on the type and extent of abnormality distribution of the individual exemplar, its cluster was labeled as one of the following 9 types: (T1) diffuse emphysema (T2) upper-lobe emphysema (T3) emphysema with early fibrosis (T4) probable NSIP (non-specific interstitial pneumonitis), (T5) confident NSIP, (T6) NSIP with concurrent emphysema, (T7) probable UIP (usual interstitial pneumonitis), (T8) confident UIP and (T9) UIP with concurrent emphysema. The quality of clusters was evaluated by radiological review and statistical analysis (ANOSIM). 


\section{Results}

Figure 2 shows representative results of the supervised classification. The voxels in panel B are color-coded using the scheme given in Figure 1. Panel C shows the $3 \mathrm{D}$ rendering of the lobe definitions. The classifications were visually verified by a radiologist. The classifications and the lobe definitions of the individual lungs were used to compute the primal pattern distribution and subsequently the individual glyphs. Figure 3 shows a mosaic of the glyphs from all 372 CT scans. Accounting for the voxel sizes and the number of voxels classified, it is worth noting that information collated across a 1.86 meter $^{3}$ space has been captured in the $5 \times 5$ inch mosaic space. Even at this resolution, the glyphs provide a succinct overview of the entire database and highlight the ease with which the intraand inter-patient disease distribution can be pre-attentively captured. With effortless effectiveness it is easy to visualize the presence of distinct cases with Chronic Obstructive Pulmonary Disease (COPD- emphysematous glyphs with blue shades), NSIP- variable amounts of ground glass and reticular distributions and UIP- extensive reticulation and honeycombing.

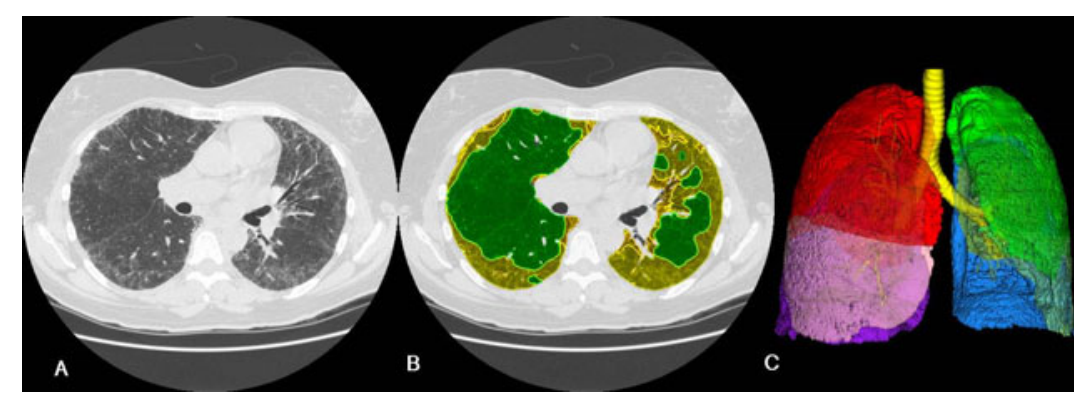

Fig. 2. Representative results of parenchymal tissue classification and 3D rendering of lobar distribution

Figure 4 shows qualitative results of the referenceless stratification. Panels A and $\mathrm{B}$ show the pair-wise similarities before and after stratification; the darker shade implies low similarity between two datasets. Affinity propagation on the original matrix (panel A) yielded 20 unique clusters. The 20 clusters correspond to the 20 diagonal sub-blocks shown (by red boxes along the diagonal) in panel $\mathrm{B}$; the stratification qualitatively reveals the maximization and minimization, respectively, of intra and inter (off-diagonal sub-blocks) cluster similarity. The maximum, minimum and mean number of candidates in the clusters were 54, 8 and 19 , respectively.

Quantitative efficacy of the stratification was examined using ANOSIM R to assess the magnitude of the differences among clusters. An $R$ value of 1 suggests that the communities completely differ among the defined groups, a value of 0 indicates no difference among groups. The pair-wise inter-cluster $\mathrm{R}$ values shown 


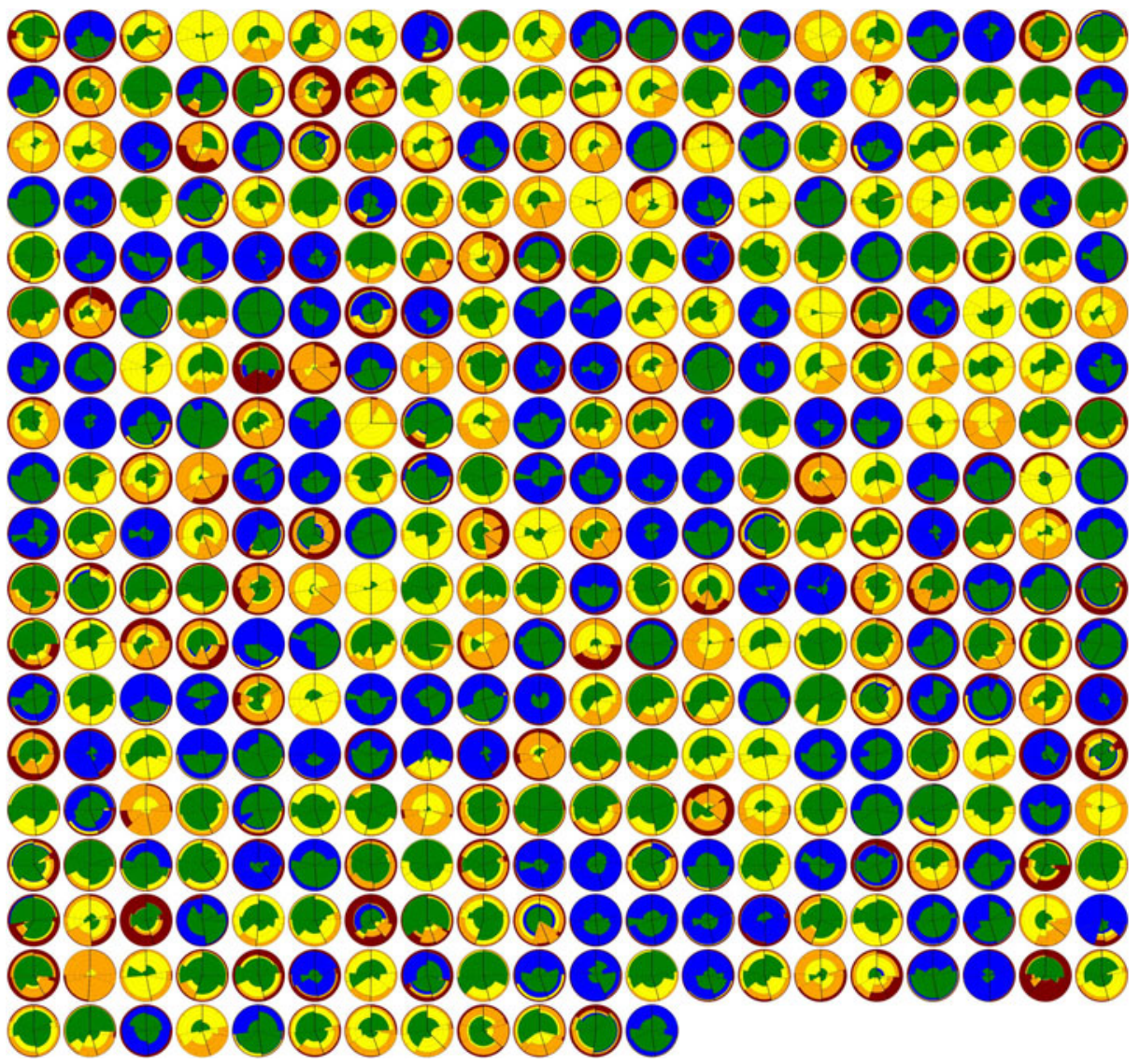

Fig. 3. Mosaic of glyphs for all 372 datasets used in this study. The glyphs provide a succinct overview of inter- and intra-subject distribution of parenchymal abnormalities.

Figure 5]A highlights the optimality of the stratification. The combined R for the 20 clusters was $0.887 \pm 0.18$ (pval $<0.0005$ ) highlighting the greater agreement of the candidates within each of the clusters. Figure $5 \mathrm{~B}$ shows the mean intra-cluster, exemplar-global and inter-exemplar distances for six representative clusters. The tightness of intra-cluster distances quantitatively validates the visual representations of stratification efficacy.

The 20 clusters were automatically categorized into clinical groups based on the abnormality distribution of their respective exemplars. Figure [6] shows the glyphs for all the exemplars, along with their clinical categories. Differentiation of the individual emphysematous lungs into upper lobe predominance (T2) has profound effect on disease management due to better outcomes after lung volume resection surgery. Though the clinical guidelines suggest nine categories, the glyphs reveal the significant pathological variations within the categories. For example, confident UIP (T8), which has poor prognosis, has five distinct 


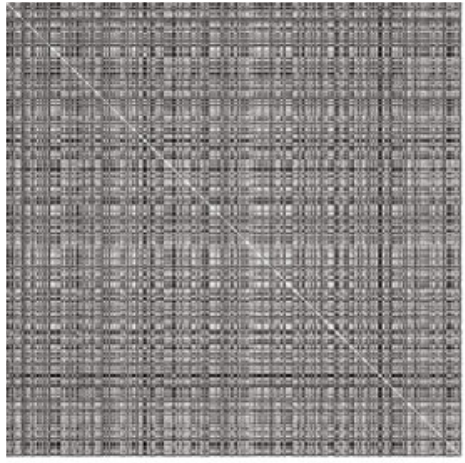

A

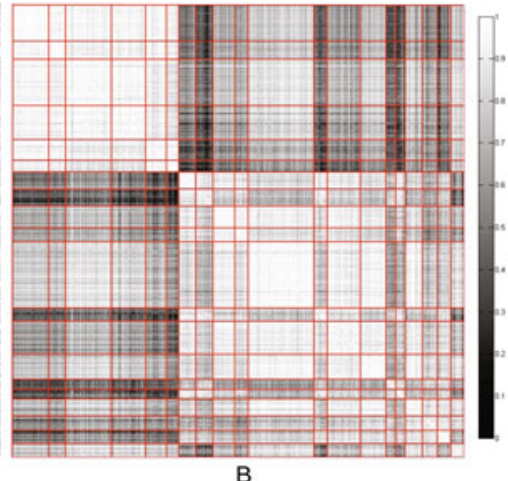

B

Fig. 4. Pairwise similarity between lungs before (A) and after (B) affinity propagation based clustering. Lighter the shade; higher the similarity.
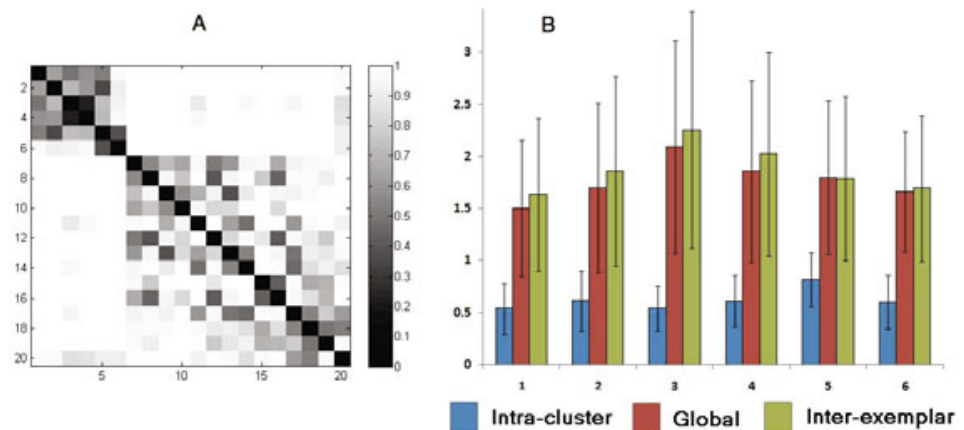

Fig. 5. Quantitative analysis of stratification showing intra-cluster pair-wise ANOSIM $R$ values (A) and comparison of intra-cluster tightness with inter-exemplar and global similarities for representative clusters (B)

variations in the regional distribution and extent of reticular and honeycombing patterns. Lumping such distinct variations into a single category could prevent the delivery of personalized and expeditious clinical care. The diagnostic disparity in performance between physicians based in academic versus community centers is well known. Such disparity is disturbing and could (at times, irreversibly) compromise patient care. The quantitative stratification proposed in this paper shows promise to reduce this discrepancy.

Figure 7 shows representative glyphs across the spectrum of diffuse pulmonary lung diseases. While the confident categories of UIP (T8) and NSIP (T5) are easily differentiable by manual radiological reviews, even after accommodating the errors due to subjective aggregation, probable categories of UIP (T7) and NSIP (T4) are often misinterpreted. The upper lobe dominance of emphysema (T2) is usually assessed using a count of voxels beyond a certain threshold. Such quantification is extremely sensitive to image slice thickness, acquisition parameters, 


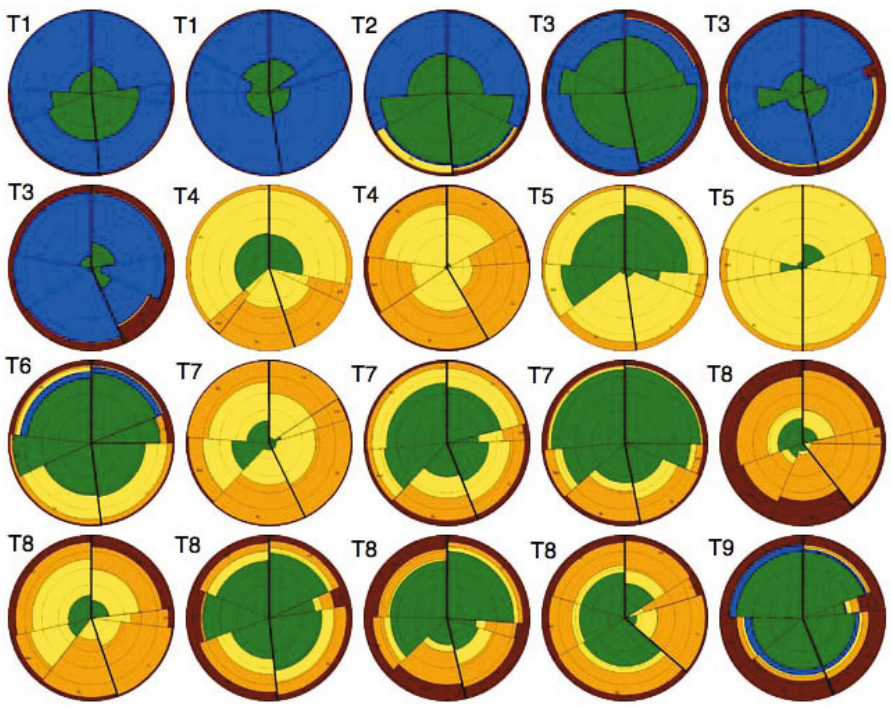

Fig. 6. The glyphs for the 20 cluster exemplars along with their categorization into clinical groups

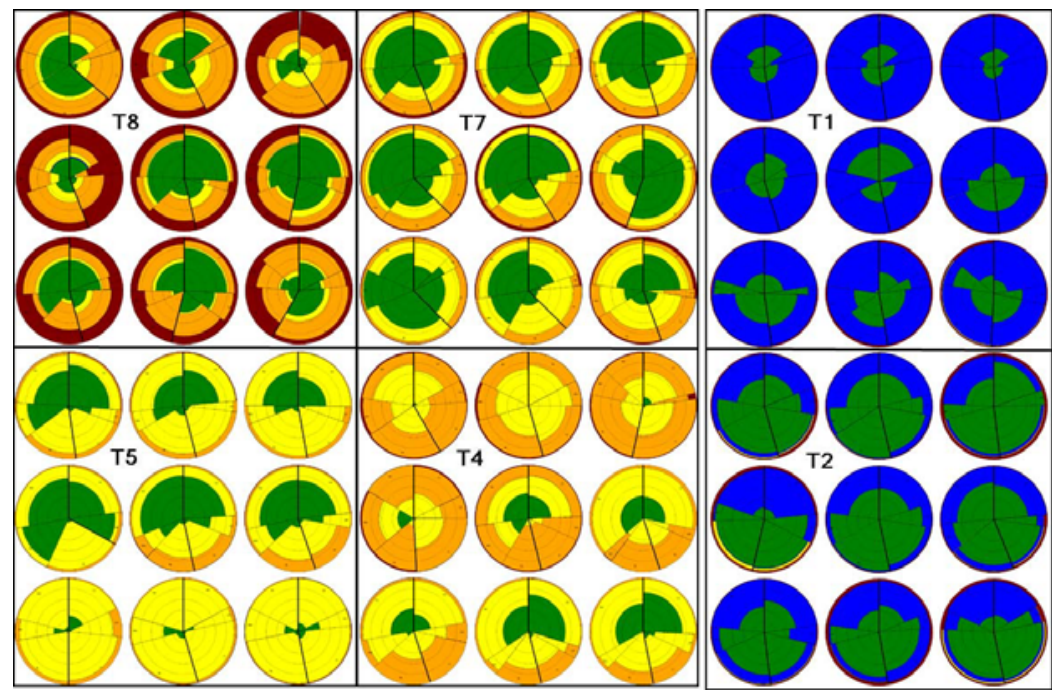

Fig. 7. Representative glyphs for the natural clusters categorized as confident UIP (T8), probable UIP (T7), Diffuse Emphysema (T1), confident NSIP (T5), probable NSIP (T4) and Upper-lobe Emphysema (T2)

and the reconstruction kernel utilized. On the other hand, the quantitative stratification and glyphs described here provide unambiguous categorization of the disease state. 


\section{Discussion and Conclusion}

Current management of diffuse parenchymal lung disease might best be described as a random walk through therapy space. However, recent advances in imaging offer opportunities to develop and validate lung-specific biomarkers as potential cross correlates to diagnosis, staging of treatment and therapy monitoring. Despite the enhanced contrast and spatial resolution of HRCT scans, classification and quantification of interstitial lung disease is difficult, and even experienced chest radiologists are challenged with differential diagnosis. Robust, expeditious and reproducible segmentation and characterization of the lung, lobes, airways, vessels and parenchymal tissues, accompanied by results summarized holistically and presented in a consistent manner, will advance the field of Computer Aided Diagnosis and elevate it to a degree of maturity and universal applicability heretofore not obtained. The stratification and visualization strategy proposed in this paper represents a major step towards harnessing the power of information technology and image computing to develop a computational framework that enables a powerful and hitherto elusive capability for pulmonary imaging evaluation: a trustable, verifiable, and clinically relevant comprehensive summary of pulmonary disease, including the extent and character of disease, both within an individual patient and across a cohort of patients.

\section{References}

1. De Craene, M., du Bois d'Aische, A., Macq, B., Warfield, S.K.: Multi-subject Registration for Unbiased Statistical Atlas Construction. In: Barillot, C., Haynor, D.R., Hellier, P. (eds.) MICCAI 2004. LNCS, vol. 3216, pp. 655-662. Springer, Heidelberg (2004)

2. Srinivasan, R., Shriram, K.S., Suryanarayanan, S.: Unbiased Stratification of Left Ventricles. In: Metaxas, D., Axel, L., Fichtinger, G., Székely, G. (eds.) MICCAI 2008, Part I. LNCS, vol. 5241, pp. 551-558. Springer, Heidelberg (2008)

3. Frey, B., Dueck, D.: Clustering by Passing Messages Between Data Points. Science 315, 972-976 (2007)

4. Akira, M., Inoue, Y., Kitaichi, M., Yamamoto, S., Arai, T., Toyokawa, K.: Usual Interstitial Pneumonia and Nonspecific Insterstitial Pneumonia with and without concurrent emphysema. Radiology 251, 271-279 (2009)

5. Ross, J.C., Estépar, R.S.J., Díaz, A., Westin, C.-F., Kikinis, R., Silverman, E.K., Washko, G.R.: Lung Extraction, Lobe Segmentation and Hierarchical Region Assessment for Quantitative Analysis on High Resolution Computed Tomography Images. In: Yang, G.-Z., Hawkes, D., Rueckert, D., Noble, A., Taylor, C. (eds.) MICCAI 2009. LNCS, vol. 5762, pp. 690-698. Springer, Heidelberg (2009) 